\title{
Banach Limit in the Stability Problem of a Linear Functional Equation
}

\author{
Roman Badora® and Janusz Brzdęk@
}

\begin{abstract}
We present some applications of the Banach limit in the study of the stability of the linear functional equation in a single variable.
\end{abstract}

Mathematics Subject Classification. 39B82, 39B52.

Keywords. Banach limit, Ulam stability problem, linear functional equation.

\section{Introduction}

Let us recall that the Banach limit is a linear functional LIM defined on the space $\ell^{\infty}$ of all bounded real sequences, satisfying the following conditions:

$$
\inf \left\{a_{n}: n \in \mathbb{N}\right\} \leq \operatorname{LIM}\left(a_{n}\right) \leq \sup \left\{a_{n}: n \in \mathbb{N}\right\}
$$

and

$$
\operatorname{LIM}\left(a_{n+k}\right)=\operatorname{LIM}\left(a_{n}\right),
$$

for all $\left(a_{n}\right) \in \ell^{\infty}$ and $k \in \mathbb{N}$ (positive integers).

The question about stability of the functional equation of group homomorphisms, formulated by S. Ulam in 1940, initiated investigations of the stability of functional equations. Currently, many studies have been published on this subject (see monographs $[8,11,12]$ or survey papers $[1,7]$ ), where the reader can learn more about this issue. The problem of stability of a given functional equation is the question whether a function satisfying that equation with a certain accuracy is close to a solution of it. In this work, we focus our attention on various forms of stability of the linear functional equation

$$
\Phi(f(x))=g(x) \Phi(x)+h(x),
$$


where $f, g, h$ are given functions and $\Phi$ is the unknown function. Special cases of (3) are the gamma functional equation

$$
\Phi(x+1)=x \Phi(x),
$$

the Schröder functional equation

$$
\Phi(f(x))=s \Phi(x),
$$

and the Abel functional equation

$$
\Phi(f(x))=\Phi(x)+1 .
$$

More information on Eq. (3) can be found in [14,15].

In this paper we consider in particular the so called iterative stability of Eq. (3) (cf. Turdza [18]). That concept of stability was introduced in 1970 by Brydak [6], who was motivated by some difficulties that he encountered in proving the classical Hyers-Ulam type stability for the equation. We show that the Banach limit technique allows us to obtain significant generalizations of Brydak's outcomes, as well as some modifications of other results. Thus we want to advertise that proof technique, which has been promoted by Badora $[2,3]$ for years.

There are known transfers of the concept of the Banach limit to the vector case (see Sofi [16]), but wishing to remain legible and to relate our results to some previously proven theorems, we remain in the scalar case.

Let us yet mention that the first classical stability result for (3) was proved by Baker [4] (with a technique based on the Banach fixed point theorem). Generalizations of Baker's outcome have been provided by Kim [13, Theorems 2.1 and 3.1] (for a simplified version of (3)) and next improved by Trif [17, Theorem 2.1] (see also [9] for some related considerations).

In the sequel $X$ always denotes a nonempty set and $f: X \rightarrow X, g, h$ : $X \rightarrow \mathbb{R}$ are given functions, unless clearly stated otherwise.

\section{Ulam Stability}

In this section, we first show that by the Banach limit method we can obtain a modification of the Trif outcome [17, Theorem 2.1]. Next we provide a slightly simplified version of the original proof of [17, Theorem 2.1]. We also state some observations concerning existence of solutions to (3) (see Remark 2).

As we have already mentioned, to be more readable, we confine ourselves to the case where functions only take the scalar values.

We start with the following generalization of [17, Theorem 2.1].

Theorem 1. Let $g(X) \subset(0, \infty), \varepsilon_{1}, \varepsilon_{2}: X \rightarrow \mathbb{R}$ and

$$
\gamma_{k}(x)=\sum_{j=0}^{\infty} \frac{\varepsilon_{k}\left(f^{j}(x)\right)}{\prod_{i=0}^{j} g\left(f^{i}(x)\right)}
$$


be finite for all $x \in X$ and $k=1,2$. Suppose that $\phi: X \rightarrow \mathbb{R}$ satisfies

$$
\varepsilon_{1}(x) \leq \phi(f(x))-g(x) \phi(x)-h(x) \leq \varepsilon_{2}(x), \quad x \in X .
$$

Then there exists a unique solution $\Phi: X \rightarrow \mathbb{R}$ of Eq. (3) such that

$$
\gamma_{1}(x) \leq \Phi(x)-\phi(x) \leq \gamma_{2}(x), \quad x \in X .
$$

Proof. Let us write the assumed inequality (5) as

$$
\varepsilon_{1}(x)+g(x) \phi(x)+h(x) \leq \phi(f(x)) \leq \varepsilon_{2}(x)+g(x) \phi(x)+h(x), \quad x \in X .
$$

We will show inductively that

$$
\begin{aligned}
\phi(x)+\sum_{j=0}^{n-1} \frac{\left.\varepsilon_{1}\left(f^{j}(x)\right)\right)}{\prod_{i=0}^{j} g\left(f^{i}(x)\right)} & \leq \frac{\phi\left(f^{n}(x)\right)}{\prod_{i=0}^{n-1} g\left(f^{i}(x)\right)}-\sum_{j=0}^{n-1} \frac{h\left(f^{j}(x)\right)}{\prod_{i=0}^{j} g\left(f^{i}(x)\right)} \\
& \leq \phi(x)+\sum_{j=0}^{n-1} \frac{\left.\varepsilon_{2}\left(f^{j}(x)\right)\right)}{\prod_{i=0}^{j} g\left(f^{i}(x)\right)}
\end{aligned}
$$

for all $x \in X$ and $n \in \mathbb{N}$ (positive integers).

To this end observe first that the case $n=1$ is just (7). So, assuming that inequalities hold for $n$, we are to prove that they are true for $n+1$. Putting $f^{n}(x)$ instead of $x$ in (7) we get

$$
\begin{aligned}
& \varepsilon_{1}\left(f^{n}(x)\right)+g\left(f^{n}(x)\right) \phi\left(f^{n}(x)\right)+h\left(f^{n}(x)\right) \leq \phi\left(f^{n+1}(x)\right) \\
& \quad \leq \varepsilon_{2}\left(f^{n}(x)\right)+g\left(f^{n}(x)\right) \phi\left(f^{n}(x)\right)+h\left(f^{n}(x)\right),
\end{aligned}
$$

which when divided by $\prod_{i=0}^{n} g\left(f^{i}(x)\right)>0$ gives

$$
\begin{aligned}
& \frac{\varepsilon_{1}\left(f^{n}(x)\right)}{\prod_{i=0}^{n} g\left(f^{i}(x)\right)}+\frac{\phi\left(f^{n}(x)\right)}{\prod_{i=0}^{n-1} g\left(f^{i}(x)\right)}+\frac{h\left(f^{n}(x)\right)}{\prod_{i=0}^{n} g\left(f^{i}(x)\right)} \leq \frac{\phi\left(f^{n+1}(x)\right)}{\prod_{i=0}^{n} g\left(f^{i}(x)\right)} \\
& \quad \leq \frac{\varepsilon_{2}\left(f^{n}(x)\right)}{\prod_{i=0}^{n} g\left(f^{i}(x)\right)}+\frac{\phi\left(f^{n}(x)\right)}{\prod_{i=0}^{n-1} g\left(f^{i}(x)\right)}+\frac{h\left(f^{n}(x)\right)}{\prod_{i=0}^{n} g\left(f^{i}(x)\right)},
\end{aligned}
$$

for all $x \in X$. Using the assumed estimates for the expression $\frac{\phi\left(f^{n}(x)\right)}{\prod_{i=0}^{n-1} g\left(f^{i}(x)\right)}$, we conclude that the inequalities are true for $n+1$.

For $x \in X$ the sequences $\left(\sum_{j=0}^{n-1} \frac{\left.\varepsilon_{1}\left(f^{j}(x)\right)\right)}{\prod_{i=0}^{j} g\left(f^{i}(x)\right)}\right)_{n \in \mathbb{N}}$ and $\left(\sum_{j=0}^{n-1} \frac{\left.\varepsilon_{2}\left(f^{j}(x)\right)\right)}{\prod_{i=0}^{j} g\left(f^{i}(x)\right)}\right)_{n \in \mathbb{N}}$ as convergent are bounded. So, (8) means that the sequence

$$
\left(\frac{\phi\left(f^{n}(x)\right)}{\prod_{i=0}^{n-1} g\left(f^{i}(x)\right)}-\sum_{k=0}^{n-1} \frac{h\left(f^{k}(x)\right)}{\prod_{j=0}^{k} g\left(f^{j}(x)\right)}\right)_{n \in \mathbb{N}}
$$

is bounded for all $x \in X$ and we can define the function $\Phi: X \rightarrow \mathbb{R}$ by

$$
\Phi(x)=\operatorname{LIM}\left(\left(\frac{\phi\left(f^{n}(x)\right)}{\prod_{i=0}^{n-1} g\left(f^{i}(x)\right)}-\sum_{k=0}^{n-1} \frac{h\left(f^{k}(x)\right)}{\prod_{j=0}^{k} g\left(f^{j}(x)\right)}\right)_{n \in \mathbb{N}}\right), \quad x \in X,
$$


where LIM is the Banach limit. that

It is well known that conditions (1) and (2) of the LIM functional imply

$$
\begin{aligned}
& \liminf _{n \rightarrow \infty}\left(\frac{\phi\left(f^{n}(x)\right)}{\prod_{i=0}^{n-1} g\left(f^{i}(x)\right)}-\sum_{k=0}^{n-1} \frac{h\left(f^{k}(x)\right)}{\prod_{j=0}^{k} g\left(f^{j}(x)\right)}\right) \leq \Phi(x) \\
& \quad \leq \limsup _{n \rightarrow \infty}\left(\frac{\phi\left(f^{n}(x)\right)}{\prod_{i=0}^{n-1} g\left(f^{i}(x)\right)}-\sum_{k=0}^{n-1} \frac{h\left(f^{k}(x)\right)}{\prod_{j=0}^{k} g\left(f^{j}(x)\right)}\right)
\end{aligned}
$$

for all $x \in X$, whence inequality (8) makes the function $\Phi$ fulfill (6). Next, using shift-invariance (2), the linearity of the Banach limit and condition (1) we get

$$
\begin{aligned}
\Phi(f(x)) & =\operatorname{LIM}\left(\left(\frac{\phi\left(f^{n+1}(x)\right)}{\prod_{i=1}^{n} g\left(f^{i}(x)\right)}-\sum_{k=1}^{n} \frac{h\left(f^{k}(x)\right)}{\prod_{j=1}^{k} g\left(f^{j}(x)\right)}\right)_{n \in \mathbb{N}}\right) \\
& =g(x) \operatorname{LIM}\left(\left(\frac{\phi\left(f^{n+1}(x)\right)}{\prod_{i=0}^{n} g\left(f^{i}(x)\right)}-\sum_{k=0}^{n} \frac{h\left(f^{k}(x)\right)}{\prod_{j=0}^{k} g\left(f^{j}(x)\right)}\right)_{n \in \mathbb{N}}\right)+h(x) \\
& =g(x) \Phi(x)+h(x), \quad x \in X,
\end{aligned}
$$

which means that $\Phi$ is a solution to (3).

For the proof of the uniqueness of $\Phi$ suppose that $\Phi_{0}: X \rightarrow \mathbb{R}$ is such that

$$
\Phi_{0}(f(x))=g(x) \Phi_{0}(x)+h(x), \quad x \in X,
$$

and

$$
\gamma_{1}(x) \leq \Phi_{0}(x)-\phi(x) \leq \gamma_{2}(x), \quad x \in X
$$

Then

$$
\begin{aligned}
\gamma_{1}(x)-\gamma_{2}(x) \leq \Phi_{0}(x)-\Phi(x) & =\left(\Phi_{0}(x)-\phi(x)\right)+(\phi(x)-\Phi(x)) \\
& \leq \gamma_{2}(x)-\gamma_{1}(x), \quad x \in X,
\end{aligned}
$$

whence

$$
\left|\Phi_{0}(x)-\Phi(x)\right| \leq \gamma_{2}(x)-\gamma_{1}(x), \quad x \in X .
$$

We show by induction that, for each $n \in \mathbb{N}_{0}:=\mathbb{N} \cup\{0\}$,

$$
\left|\Phi_{0}(x)-\Phi(x)\right| \leq \sum_{j=n}^{\infty} \frac{\varepsilon_{2}\left(f^{j}(x)\right)}{\prod_{i=0}^{j}\left|g\left(f^{i}(x)\right)\right|}-\sum_{j=n}^{\infty} \frac{\varepsilon_{1}\left(f^{j}(x)\right)}{\prod_{i=0}^{j}\left|g\left(f^{i}(x)\right)\right|}, \quad x \in X .
$$

Clearly, the case $n=0$ is just (11). So, assume that (12) holds for a fixed $n \in \mathbb{N}_{0}$. Then replacing $x$ by $f(x)$ in (11), by (3) and (9), for each $x \in X$ we obtain 


$$
|g(x)|\left|\Phi_{0}(x)-\Phi(x)\right|=\left|\Phi_{0}(f(x))-\Phi(f(x))\right| \leq \sum_{j=n}^{\infty} \frac{\varepsilon_{2}\left(f^{j+1}(x)\right)-\varepsilon_{1}\left(f^{j+1}(x)\right)}{\prod_{i=0}^{j}\left|g\left(f^{i+1}(x)\right)\right|},
$$

which implies that

$$
\left|\Phi_{0}(x)-\Phi(x)\right| \leq \sum_{j=n}^{\infty} \frac{\varepsilon_{2}\left(f^{j+1}(x)\right)-\varepsilon_{1}\left(f^{j+1}(x)\right)}{\prod_{i=0}^{j+1}\left|g\left(f^{i}(x)\right)\right|}=\sum_{j=n+1}^{\infty} \frac{\varepsilon_{2}\left(f^{j}(x)\right)-\varepsilon_{1}\left(f^{j}(x)\right)}{\prod_{i=0}^{j}\left|g\left(f^{i}(x)\right)\right|} .
$$

Thus we have proved that (12) is valid for each $n \in \mathbb{N}_{0}$, whence letting $n \rightarrow \infty$ we obtain $\Phi_{0}=\Phi$, which ends the proof.

Note that a very particular case of Theorem 1 (with $\varepsilon_{1}=\varepsilon_{2}$ ) is the following simple observation.

Corollary 1. Let $g(X) \subset(0, \infty), \varepsilon: X \rightarrow \mathbb{R}$ and

$$
\gamma(x)=\sum_{j=0}^{\infty} \frac{\varepsilon\left(f^{j}(x)\right)}{\prod_{i=0}^{j}\left|g\left(f^{i}(x)\right)\right|}<\infty, \quad x \in X .
$$

Then a function $\phi: X \rightarrow \mathbb{R}$ satisfies

$$
\phi(f(x))=g(x) \phi(x)+h(x)+\varepsilon(x), \quad x \in X,
$$

if and only if there exists a solution $\Phi: X \rightarrow \mathbb{R}$ of Eq. (3) such that

$$
\phi(x)=\Phi(x)-\gamma(x), \quad x \in X .
$$

Proof. The necessary condition of the statement follows from Theorem 1 with $\varepsilon_{1}=\varepsilon_{2}=\varepsilon$. The converse is easy to verify.

If $\varepsilon_{1}=-\varepsilon_{2}$ in Theorem 1 , then we get the scalar version of [17, Theorem 2.1 , i.e., the next theorem (but only in the case $g(X) \subset(0, \infty)$ ). We prove Theorem 2 in a somewhat different way than in [17], i.e., by the Banach limit method. Note that the assumption $g(X) \subset(0, \infty)$ has been replaced in Theorem 2 by $0 \notin g(X)$; moreover, the uniqueness statement has been strengthened a bit.

Theorem 2. Let $0 \notin g(X)$ and $\varepsilon: X \rightarrow[0, \infty)$ be such that (13) holds. Suppose that $\phi: X \rightarrow \mathbb{R}$ satisfies

$$
|\phi(f(x))-g(x) \phi(x)-h(x)| \leq \varepsilon(x), \quad x \in X .
$$

Then there exists a unique solution $\Phi: X \rightarrow \mathbb{R}$ of Eq. (3) with

$$
|\Phi(x)-\phi(x)| \leq \gamma(x), \quad x \in X
$$

Moreover, $\Phi$ is given by

$$
\Phi(x)=\operatorname{LIM}\left(\left(\frac{\phi\left(f^{n}(x)\right)}{\prod_{i=0}^{n-1} g\left(f^{i}(x)\right)}-\sum_{k=0}^{n-1} \frac{h\left(f^{k}(x)\right)}{\prod_{j=0}^{k} g\left(f^{j}(x)\right)}\right)_{n \in \mathbb{N}}\right), \quad x \in X,(16)
$$


where LIM denotes the Banach limit and it is the only solution to (3) such that

$$
\sup _{x \in X} \frac{|\Phi(x)-\phi(x)|}{\gamma(x)}<\infty .
$$

Proof. First we show inductively that

$$
\left|\frac{\phi\left(f^{n}(x)\right)}{\prod_{i=0}^{n-1} g\left(f^{i}(x)\right)}-\phi(x)-\sum_{k=0}^{n-1} \frac{h\left(f^{k}(x)\right)}{\prod_{j=0}^{k} g\left(f^{j}(x)\right)}\right| \leq \sum_{j=0}^{n-1} \frac{\varepsilon\left(f^{j}(x)\right)}{\prod_{i=0}^{j}\left|g\left(f^{i}(x)\right)\right|}
$$

for all $x \in X$ and $n \geq 1$.

Clearly, the case $n=1$ is just (14) divided by $|g(x)|$. So, assume that the inequality is valid for a fixed $n \geq 1$. We are to prove that this is the case for $n+1$.

Replacing $x$ by $f^{n}(x)$ in (14) and dividing by $\prod_{i=0}^{n} g\left(f^{i}(x)\right) \neq 0$ we get

$$
\left|\frac{\phi\left(f^{n+1}(x)\right)}{\prod_{i=0}^{n} g\left(f^{i}(x)\right)}-\frac{\phi\left(f^{n}(x)\right)}{\prod_{i=0}^{n-1} g\left(f^{i}(x)\right)}-\frac{h\left(f^{n}(x)\right)}{\prod_{i=0}^{n} g\left(f^{i}(x)\right)}\right| \leq \frac{\varepsilon\left(f^{n}(x)\right)}{\prod_{i=0}^{n}\left|g\left(f^{i}(x)\right)\right|}, \quad x \in X,
$$

whence and by the assumed inequality (18), for all $x \in X$ we get

$$
\begin{aligned}
& \left|\frac{\phi\left(f^{n+1}(x)\right)}{\prod_{i=0}^{n} g\left(f^{i}(x)\right)}-\phi(x)-\sum_{k=0}^{n} \frac{h\left(f^{k}(x)\right)}{\prod_{j=0}^{k} g\left(f^{j}(x)\right)}\right| \\
& \quad \leq\left|\frac{\phi\left(f^{n}(x)\right)}{\prod_{i=0}^{n-1} g\left(f^{i}(x)\right)}-\phi(x)\right|+\frac{\varepsilon\left(f^{n}(x)\right)}{\prod_{i=0}^{n}\left|g\left(f^{i}(x)\right)\right|} \\
& \quad \leq \sum_{j=0}^{n-1} \frac{\varepsilon\left(f^{j}(x)\right)}{\prod_{i=0}^{j}\left|g\left(f^{i}(x)\right)\right|}+\frac{\varepsilon\left(f^{n}(x)\right)}{\prod_{i=0}^{n}\left|g\left(f^{i}(x)\right)\right|}=\sum_{j=0}^{n} \frac{\varepsilon\left(f^{j}(x)\right)}{\prod_{i=0}^{j}\left|g\left(f^{i}(x)\right)\right|} .
\end{aligned}
$$

Thus we have proved that (18) holds for every $n \in \mathbb{N}$, which means that, for every $x \in X$,

$$
-\gamma(x)+\phi(x) \leq \frac{\phi\left(f^{n+1}(x)\right)}{\prod_{i=0}^{n} g\left(f^{i}(x)\right)}-\sum_{k=0}^{n} \frac{h\left(f^{k}(x)\right)}{\prod_{j=0}^{k} g\left(f^{j}(x)\right)} \leq \phi(x)+\gamma(x)
$$

and consequently the sequence

$$
\left(\frac{\phi\left(f^{n}(x)\right)}{\prod_{i=0}^{n-1} g\left(f^{i}(x)\right)}-\sum_{k=0}^{n-1} \frac{h\left(f^{k}(x)\right)}{\prod_{j=0}^{k} g\left(f^{j}(x)\right)}\right)_{n \in \mathbb{N}}
$$

is bounded.

So, we can define the function $\Phi: X \rightarrow \mathbb{R}$ by (16).

The normalization condition (1) of the LIM functional and inequalities (19) make the function $\Phi$ fulfill (15). 
Next, using the linearity of the Banach limit, condition (1) and finally the shift-invariance (2), we get

$$
\begin{aligned}
\Phi(f(x)) & =\operatorname{LIM}\left(\left(\frac{\phi\left(f^{n+1}(x)\right)}{\prod_{i=1}^{n} g\left(f^{i}(x)\right)}-\sum_{k=1}^{n} \frac{h\left(f^{k}(x)\right)}{\prod_{j=1}^{k} g\left(f^{j}(x)\right)}\right)_{n \in \mathbb{N}}\right) \\
& =g(x) \operatorname{LIM}\left(\left(\frac{\phi\left(f^{n+1}(x)\right)}{\prod_{i=0}^{n} g\left(f^{i}(x)\right)}-\sum_{k=0}^{n} \frac{h\left(f^{k}(x)\right)}{\prod_{j=0}^{k} g\left(f^{j}(x)\right)}\right)_{n \in \mathbb{N}}\right)+h(x) \\
& =g(x) \Phi(x)+h(x), \quad x \in X
\end{aligned}
$$

which means that $\Phi$ is a solution to (3).

It remains to show the uniqueness of $\Phi$. So, assume that also $\Psi: X \rightarrow \mathbb{R}$ satisfies the equation

$$
\Psi(f(x))=g(x) \Psi(x)+h(x), \quad x \in X,
$$

and there is a $\mu>0$ such that

$$
\sup _{x \in X} \frac{|\Psi(x)-\phi(x)|}{\gamma(x)}<\mu .
$$

Then

$$
|\Phi(x)-\Psi(x)| \leq \mu_{0} \gamma(x), \quad x \in X,
$$

where $\mu_{0}:=\mu+1$. We show by induction that, for each $n \in \mathbb{N}_{0}$,

$$
|\Phi(x)-\Psi(x)| \leq \mu_{0} \sum_{j=n}^{\infty} \frac{\varepsilon\left(f^{j}(x)\right)}{\prod_{i=0}^{j}\left|g\left(f^{i}(x)\right)\right|}, \quad x \in X .
$$

Clearly, the case $n=0$ is just (23). Assume that (24) holds for a fixed $n \in \mathbb{N}_{0}$. Then replacing $x$ by $f(x)$ in (24), by (21), we obtain

$$
|g(x)||\Phi(x)-\Psi(x)|=|\Phi(f(x))-\Psi(f(x))| \leq \mu_{0} \sum_{j=n}^{\infty} \frac{\varepsilon\left(f^{j+1}(x)\right)}{\prod_{i=0}^{j}\left|g\left(f^{i+1}(x)\right)\right|}, \quad x \in X,
$$

which implies that

$$
|\Phi(x)-\Psi(x)| \leq \mu_{0} \sum_{j=n}^{\infty} \frac{\varepsilon\left(f^{j+1}(x)\right)}{\prod_{i=0}^{j+1}\left|g\left(f^{i}(x)\right)\right|}=\mu_{0} \sum_{j=n+1}^{\infty} \frac{\varepsilon\left(f^{j}(x)\right)}{\prod_{i=0}^{j}\left|g\left(f^{i}(x)\right)\right|}, \quad x \in X .
$$

Thus we have proved that (24) is valid for each $n \in \mathbb{N}_{0}$, whence letting $n \rightarrow \infty$ we obtain $\Phi=\Psi$. This ends the proof.

Remark 1. The statement on the uniqueness of $\Phi$ in Theorem 2 can be strengthened a bit. Namely, $\Phi$ is the only solution to (3) satisfying the inequality

$$
\frac{|\Phi(x)-\phi(x)|}{\gamma(x)} \leq \eta(x), \quad x \in X
$$


with some function $\eta: X \rightarrow[0, \infty)$ such that the sequence $\left(\eta\left(f^{n}(x)\right)\right)_{n \in \mathbb{N}_{0}}$ is bounded for each $x \in X$. It is very easy to modify the end of Theorem 2 proof accordingly [in particular, replacing $\mu_{0}$ by $\eta\left(f^{n}(x)\right)$ in $(24)$ ].

The following observation concerning solutions to Eq. (3) can be easily deduced from the proof of Theorem 2. It shows further advantages of using the Banach limit (see also Remark 4).

Remark 2. Let $0 \notin g(X)$ and $\phi: X \rightarrow \mathbb{R}$ be such that the sequence

$$
\kappa_{n}(x):=\frac{\phi\left(f^{n}(x)\right)}{\prod_{i=0}^{n-1} g\left(f^{i}(x)\right)}-\sum_{k=0}^{n-1} \frac{h\left(f^{k}(x)\right)}{\prod_{j=0}^{k} g\left(f^{j}(x)\right)}, \quad n \in \mathbb{N},
$$

is bounded. Then we can define a function $\Phi: X \rightarrow \mathbb{R}$ by (16) and (20) shows that $\Phi$ is a solution to (3).

On the other hand, (18) with $\varepsilon(x) \equiv 0$ shows that every solution of $\Phi: X \rightarrow \mathbb{R}$ of (3) fulfils the condition

$$
\Phi(x)=\frac{\Phi\left(f^{n}(x)\right)}{\prod_{i=0}^{n-1} g\left(f^{i}(x)\right)}-\sum_{k=0}^{n-1} \frac{h\left(f^{k}(x)\right)}{\prod_{j=0}^{k} g\left(f^{j}(x)\right)}, \quad x \in X .
$$

Thus we obtain a conclusion that Eq. (3) has at least one solution if and only if there exists a function $\phi: X \rightarrow \mathbb{R}$ such that the sequence $\left(\kappa_{n}(x)\right)_{n \in \mathbb{N}}$ is bounded for each $x \in X$.

The next two remarks show that assumption (13) of Theorem 2 cannot be omitted.

Remark 3. If $X \subset \mathbb{R}, f(x)=x$ and $g(x)=1$ for $x \in X$, and $h$ is a non-zero bounded function, then for every function $\phi: X \rightarrow \mathbb{R}$ we have

$$
|\phi(x)-\phi(x)-h(x)| \leq \varepsilon_{0}, \quad x \in X,
$$

where $\varepsilon_{0}=\sup _{x \in X}|h(x)|$, but the set of solutions of the equation

$$
\Phi(x)=\Phi(x)+h(x), \quad x \in X
$$

is empty.

Remark 4. Assume that $0 \notin g(X)$ and there is an $x_{0} \in X$ such that

$$
f^{2}\left(x_{0}\right)=x_{0}, \quad g\left(x_{0}\right)=2, \quad g\left(f\left(x_{0}\right)\right)=\frac{1}{2}, \quad h\left(x_{0}\right) h\left(f\left(x_{0}\right)\right)>0 .
$$

Every function $\phi: X \rightarrow \mathbb{R}$ satisfies

$$
|\phi(f(x))-g(x) \phi(x)-h(x)| \leq \varepsilon(x), \quad x \in X,
$$

with $\varepsilon(x)=|\phi(f(x))-g(x) \phi(x)-h(x)|$.

On the other hand, according to $(27), \prod_{j=0}^{k} g\left(f^{j}\left(x_{0}\right)\right) \in\{1,2\}$ for all $k \in$ $\mathbb{N}$ and the sequence $\left(\phi\left(f^{n}\left(x_{0}\right)\right)\right)_{n \in \mathbb{N}}$ is bounded for each function $\phi: X \rightarrow \mathbb{R}$. Consequently

$$
\lim _{n \rightarrow \infty}\left|\kappa_{n}\left(x_{0}\right)\right|=\infty
$$


for every $\phi: X \rightarrow \mathbb{R}$, where $\kappa_{n}(x)$ is defined by (26). This means that Eq. (3) does not have any solution $\Phi: X \rightarrow \mathbb{R}$ (see the conclusion of Remark 2).

The next remark provides an example of a very simple application of Theorem 2.

Remark 5. If $\alpha \in(1, \infty)$ and $g(x)=\alpha$ for $x \in X$, then

$$
\gamma(x)=\sum_{j=0}^{\infty} \prod_{i=0}^{j} \frac{1}{g\left(f^{i}(x)\right)}=\sum_{j=1}^{\infty} \frac{1}{\alpha^{j}}=\frac{1}{\alpha-1}, \quad x \in X,
$$

and, by Theorem 2, for every function $\phi: X \rightarrow \mathbb{R}$ fulfilling

$$
|\phi(f(x))-\alpha \phi(x)| \leq \varepsilon, \quad x \in X,
$$

there exists a unique solution $\Phi: X \rightarrow \mathbb{R}$ of the equation

$$
\Phi(f(x))=\alpha \Phi(x)
$$

such that

$$
|\phi(x)-\Phi(x)| \leq \frac{\varepsilon}{\alpha-1}, \quad x \in X .
$$

As Forti [10] noted, in the case where $(X,+)$ is a commutative semigroup, $\alpha=2$ and $f(x)=2 x$, this result allows us to obtain the classical stability result for the Cauchy equation (see also [5, pp. 11-14]). Analogously, we may obtain the stability of the quadratic functional equation and several other similar equations.

\section{Iterative Stability}

As we have already mentioned, Brydak [6] introduced the concept of stability, which was later referred to as iterative stability (see Turdza [18]). Below we show that the Banach limit method allows to prove the following two generalizations of Brydak's theorem (but without the regularity properties).

Theorem 3. Assume that $f$ is bijective and $0 \notin g(X)$. If a function $\phi: X \rightarrow \mathbb{R}$ satisfies inequality

$$
\left|\phi\left(f^{n}(x)\right)-G_{n}(x) \phi(x)-G_{n}(x) \sum_{i=0}^{n-1} \frac{h\left(f^{i}(x)\right)}{G_{i+1}(x)}\right| \leq \varepsilon,
$$

for all $x \in X$ and $n \in \mathbb{N}$, where

$$
G_{n}(x):=\prod_{i=0}^{n-1} g\left(f^{i}(x)\right), \quad x \in X, \quad n \in \mathbb{N},
$$

then there exists a solution $\Phi: X \rightarrow \mathbb{R}$ of Eq. (3) such that

$$
|\Phi(x)-\phi(x)| \leq \varepsilon, \quad x \in X .
$$


Proof. First we observe that, for all $x \in X$ and $n \in \mathbb{N}$,

$$
\begin{aligned}
G_{n}(x) \sum_{i=0}^{n-1} \frac{h\left(f^{i}(x)\right)}{G_{i+1}(x)} & =G_{n}(x) \sum_{i=0}^{n-2} \frac{h\left(f^{i}(x)\right)}{G_{i+1}(x)}+G_{n}(x) \frac{h\left(f^{n-1}(x)\right)}{G_{n}(x)} \\
& =\sum_{i=0}^{n-2} h\left(f^{i}(x)\right) \prod_{j=i+1}^{n-1} g\left(f^{j}(x)\right)+h\left(f^{n-1}(x)\right),
\end{aligned}
$$

whence by our assumption (28),

$$
\left|\phi\left(f^{n}(x)\right)-G_{n}(x) \phi(x)-\sum_{i=0}^{n-2} h\left(f^{i}(x)\right) \prod_{j=i+1}^{n-1} g\left(f^{j}(x)\right)-h\left(f^{n-1}(x)\right)\right| \leq \varepsilon .
$$

If we replace in the last inequality $x$ by $f^{-n}(x)$, then we obtain

$$
\begin{aligned}
& \mid-\phi(x)+\phi\left(f^{-n}(x)\right) \prod_{i=0}^{n-1} g\left(f^{-n+i}(x)\right) \\
& +\sum_{i=0}^{n-2} h\left(f^{-n+i}(x)\right) \prod_{j=i+1}^{n-1} g\left(f^{-n+j}(x)\right)+h\left(f^{-1}(x)\right) \mid \leq \varepsilon
\end{aligned}
$$

for all $x \in X$ and $n \in \mathbb{N}$. But, for all $x \in X$ and $n \in \mathbb{N}$,

$$
\begin{aligned}
\sum_{i=0}^{n-2} h\left(f^{-n+i}(x)\right) \prod_{j=i+1}^{n-1} g\left(f^{-n+j}(x)\right) & =\sum_{i=0}^{n-2} h\left(f^{-n+i}(x)\right) \prod_{j=-n+i+1}^{-1} g\left(f^{j}(x)\right) \\
& =\sum_{i=2}^{n} h\left(f^{-i}(x)\right) \prod_{j=1}^{i-1} g\left(f^{-j}(x)\right)
\end{aligned}
$$

whence (31) can be rewritten as

$$
\begin{aligned}
& \mid-\phi(x)+\phi\left(f^{-n}(x)\right) \prod_{i=1}^{n} g\left(f^{-i}(x)\right) \\
& \quad+\sum_{i=2}^{n} h\left(f^{-i}(x)\right) \prod_{j=1}^{i-1} g\left(f^{-j}(x)\right)+h\left(f^{-1}(x)\right) \mid \leq \varepsilon .
\end{aligned}
$$

Therefore,

$$
\begin{aligned}
-\varepsilon+\phi(x) \leq & \phi\left(f^{-n}(x)\right) \prod_{i=1}^{n} g\left(f^{-i}(x)\right) \\
& +\sum_{i=2}^{n} h\left(f^{-i}(x)\right) \prod_{j=1}^{i-1} g\left(f^{-j}(x)\right)+h\left(f^{-1}(x)\right) \leq \phi(x)+\varepsilon
\end{aligned}
$$


for all $x \in X$ and $n \in \mathbb{N}$, which means that the sequence $\left(a_{n}(x)\right)_{n \in \mathbb{N}}$ defined, for a fixed $x \in X$, by

$$
a_{n}(x)=\phi\left(f^{-n}(x)\right) \prod_{i=1}^{n} g\left(f^{-i}(x)\right)+\sum_{i=2}^{n} h\left(f^{-i}(x)\right) \prod_{j=1}^{i-1} g\left(f^{-j}(x)\right)+h\left(f^{-1}(x)\right),
$$

is bounded (belongs to the space $\ell^{\infty}$ ).

So, we may define a function $\Phi: X \rightarrow \mathbb{R}$ by the following formulae

$$
\Phi(x)=\operatorname{LIM}\left(\left(a_{n}(x)\right)_{n \in \mathbb{N}}\right), \quad x \in X .
$$

Clearly, $\Phi$ satisfies inequality (30). Next, observe that, for all $x \in X$ and $n \in \mathbb{N}$,

$$
\begin{aligned}
a_{n}\left(f^{-1}(x)\right)= & \phi\left(f^{-(n+1)}(x)\right) \prod_{i=1}^{n} g\left(f^{-(i+1)}(x)\right) \\
& +\sum_{i=2}^{n} h\left(f^{-(i+1)}(x)\right) \prod_{j=1}^{i-1} g\left(f^{-(j+1)}(x)\right)+h\left(f^{-2}(x)\right) \\
= & \phi\left(f^{-(n+1)}(x)\right) \prod_{i=2}^{n+1} g\left(f^{-i}(x)\right) \\
& +\sum_{i=2}^{n} h\left(f^{-(i+1)}(x)\right) \prod_{j=2}^{i} g\left(f^{-j}(x)\right)+h\left(f^{-2}(x)\right) \\
= & \phi\left(f^{-(n+1)}(x)\right) \prod_{i=2}^{n+1} g\left(f^{-i}(x)\right) \\
& +\sum_{i=3}^{n+1} h\left(f^{-i}(x)\right) \prod_{j=2}^{i-1} g\left(f^{-j}(x)\right)+h\left(f^{-2}(x)\right) \\
= & \frac{\phi\left(f^{-(n+1)}(x)\right)}{g\left(f^{-1}(x)\right)} \prod_{i=1}^{n+1} g\left(f^{-i}(x)\right)+\sum_{i=3}^{n+1} h\left(f^{-i}(x)\right) \prod_{j=1}^{i-1} g\left(f^{-j}(x)\right) \\
& \left.+h\left(f^{-2}(x)\right) g\left(f^{-1}(x)\right)+h\left(f^{-1}(x)\right)\right)-\frac{h\left(f^{-1}(x)\right)}{g\left(f^{-1}(x)\right)} \\
= & \frac{1}{g\left(f^{-1}(x)\right)}\left(a_{n+1}(x)-\frac{h\left(f^{-1}(x)\right.}{g\left(f^{-1}(x)\right)}\right) .
\end{aligned}
$$

Thus, by the linearity and the invariance (2) of LIM, we get

$$
\begin{aligned}
\Phi\left(f^{-1}(x)\right) & =\operatorname{LIM}\left(\left(a_{n}\left(f^{-1}(x)\right)\right)_{n \in \mathbb{N}}\right) \\
& =\frac{1}{g\left(f^{-1}(x)\right)} \operatorname{LIM}\left(\left(a_{n+1}(x)-\frac{h\left(f^{-1}(x)\right)}{g\left(f^{-1}(x)\right)}\right)_{n \in \mathbb{N}}\right)
\end{aligned}
$$




$$
=\frac{1}{g\left(f^{-1}(x)\right)} \Phi(x)-\frac{h\left(f^{-1}(x)\right)}{g\left(f^{-1}(x)\right)} .
$$

Now, replacing $x$ by $f(x)$ we see that the function $\Phi$ satisfies Eq. (3), which ends the proof.

Remark 6. There arises a natural question concerning the uniqueness of $\Phi$ in Theorem 3. In the general case, without any additional assumptions, there is no uniqueness. For instance, if $g(x) \equiv 1$ and $h(x) \equiv 0$, then every function $\phi: X \rightarrow[0, \varepsilon]$ satisfies inequality (28) for all $x \in X$ and $n \in \mathbb{N}$ and every constant function $\Phi: X \rightarrow[0, \varepsilon]$ is a solution to Eq. (3) such that (30) holds.

The situation is somewhat different if $g(X) \neq\{1\}$. Namely, assume that the assumptions of Theorem 3 are valid and $\Phi, \Psi: X \rightarrow \mathbb{R}$ are solutions of (3). Then

$$
\begin{aligned}
& |\Phi(x)-\Psi(x)|=\frac{|\Phi(f(x))-\Psi(f(x))|}{|g(x)|}, \quad x \in X, g(x) \neq 0, \\
& |\Phi(x)-\Psi(x)|=\left|g\left(f^{-1}(x)\right)\right|\left|\Phi\left(f^{-1}(x)\right)-\Psi\left(f^{-1}(x)\right)\right|, \quad x \in X .
\end{aligned}
$$

It is easy to show by induction that

$$
\begin{gathered}
|\Phi(x)-\Psi(x)|=\frac{\left|\Phi\left(f^{n}(x)\right)-\Psi\left(f^{n}(x)\right)\right|}{\left|G_{n}(x)\right|}, \quad x \in X, n \in \mathbb{N}, G_{n}(x) \neq 0, \\
|\Phi(x)-\Psi(x)|=\left|G_{-n}(x)\right|\left|\Phi\left(f^{-n}(x)\right)-\Psi\left(f^{-n}(x)\right)\right|, \quad x \in X, n \in \mathbb{N},
\end{gathered}
$$

where

$$
G_{-n}(x):=\prod_{i=1}^{n} g\left(f^{-i}(x)\right), \quad n \in \mathbb{N}, x \in I .
$$

Now suppose that

$$
|\phi(x)-\Phi(x)| \leq \varepsilon, \quad|\phi(x)-\Psi(x)| \leq \varepsilon, \quad x \in X .
$$

Then

$$
|\Phi(x)-\Psi(x)| \leq 2 \varepsilon, \quad x \in X
$$

Consequently, for every $x \in X$ with $\sup _{n \in \mathbb{N}}\left|G_{n}(x)\right|=\infty$ and $G_{n}(x) \neq 0$ for all $n \in \mathbb{N}$, by $(32)$ we have $\Phi(x)=\Psi(x)$. The same is true, in view of (33), for every $x \in X$ with $\inf _{n \in \mathbb{N}}\left|G_{-n}(x)\right|=0$.

Theorem 4. Let $0 \notin g(X), \varepsilon: X \rightarrow[0,+\infty), G_{n}$ be given by (29), and

$$
\Gamma(x):=\liminf _{n \rightarrow \infty}\left|G_{n}(x)\right|>0, \quad x \in X .
$$

If a function $\phi: X \rightarrow \mathbb{R}$ satisfies the following inequality

$$
\left|\phi\left(f^{n}(x)\right)-G_{n}(x) \phi(x)-G_{n}(x) \sum_{i=0}^{n-1} \frac{h\left(f^{i}(x)\right)}{G_{i+1}(x)}\right| \leq \varepsilon(x)
$$


for all $x \in X$ and $n \in \mathbb{N}$, then there is a solution $\Phi: X \rightarrow \mathbb{R}$ of Eq. (3) such that

$$
|\Phi(x)-\phi(x)| \leq \frac{\varepsilon(x)}{\Gamma(x)}, \quad x \in X .
$$

Proof. Write $\Gamma_{k}(x):=\inf _{n \in \mathbb{N}}\left|G_{n+k}(x)\right|$ for $x \in X$ and $k \in \mathbb{N}_{0}$. Note that

$$
\Gamma_{k}(x)>0, \quad x \in X, k \in \mathbb{N}_{0},
$$

because $\Gamma(x)>0$ and $G_{n}(x) \neq 0$ for all $x \in X$ and $n \in \mathbb{N}$.

By our assumption

$$
\left|\frac{\phi\left(f^{n}(x)\right)}{G_{n}(x)}-\phi(x)-\sum_{i=0}^{n-1} \frac{h\left(f^{i}(x)\right)}{G_{i+1}(x)}\right| \leq \frac{\varepsilon(x)}{\left|G_{n}(x)\right|},
$$

for all $x \in X$ and $n \in \mathbb{N}$. For $n \in \mathbb{N}$ we define $\phi_{n}: X \rightarrow \mathbb{R}$ as follows

$$
\phi_{n}(x)=\frac{\phi\left(f^{n}(x)\right)}{G_{n}(x)}-\sum_{i=0}^{n-1} \frac{h\left(f^{i}(x)\right)}{G_{i+1}(x)}, \quad x \in X .
$$

Then

$$
\left|\phi_{n+k}(x)-\phi(x)\right| \leq \frac{\varepsilon(x)}{\left|G_{n+k}(x)\right|} \leq \frac{\varepsilon(x)}{\Gamma_{k}(x)}, \quad x \in X, n \in \mathbb{N}, k \in \mathbb{N}_{0},
$$

which means that

$$
\phi(x)-\frac{\varepsilon(x)}{\Gamma_{k}(x)} \leq \phi_{n+k}(x) \leq \phi(x)+\frac{\varepsilon(x)}{\Gamma_{k}(x)}, \quad x \in X, n \in \mathbb{N}, k \in \mathbb{N}_{0} .
$$

Therefore, for any $x \in X$ and $k \in \mathbb{N}_{0}$ the sequence $\left(\phi_{n+k}(x)\right)_{n \in \mathbb{N}}$ is bounded.

Let $\Phi: X \rightarrow \mathbb{R}$ be defined by

$$
\Phi(x)=\operatorname{LIM}\left(\phi_{n}(x)\right), \quad x \in X .
$$

Since $\operatorname{LIM}\left(\left(\phi_{n}(x)\right)_{n \in \mathbb{N}}\right)=\operatorname{LIM}\left(\left(\phi_{n+k}(x)\right)_{n \in \mathbb{N}}\right)$ for all $x \in X$ and $k \in \mathbb{N}$ [see (1)], by (37), we get

$$
|\Phi(x)-\phi(x)| \leq \frac{\varepsilon(x)}{\Gamma_{k}(x)}, \quad x \in X, k \in \mathbb{N}_{0} .
$$

Hence

$$
|\Phi(x)-\phi(x)| \leq \frac{\varepsilon(x)}{\sup _{k \in \mathbb{N}_{0}} \Gamma_{k}(x)}, \quad x \in X,
$$

which yields (35) (because $\Gamma(x)=\liminf _{n \rightarrow \infty}\left|G_{n}(x)\right|=\sup _{k \in \mathbb{N}_{0}} \Gamma_{k}(x)$ ).

Moreover, for every $x \in X$ and $n \in \mathbb{N}$, we have

$$
\phi_{n}(f(x))=g(x) \phi_{n+1}(x)+h(x),
$$

whence

$$
\begin{aligned}
\Phi(f(x)) & =\operatorname{LIM}\left(\left(\phi_{n}(f(x))\right)_{n \in \mathbb{N}}\right)=\operatorname{LIM}\left(\left(g(x) \phi_{n+1}(x)+h(x)\right)_{n \in \mathbb{N}}\right) \\
& =g(x) \Phi(x)+h(x)
\end{aligned}
$$


which ends the proof.

Remark 7. If in Theorem 4 we have $k:=\sup _{x \in X} \frac{1}{|\Gamma(x)|}<\infty$, then (35) implies that $|\Phi(x)-\phi(x)| \leq k \varepsilon(x)$ for $x \in X$, which corresponds to the conclusion of Brydak's theorem.

The reasoning of Remark 6 concerning the uniqueness of $\Phi$ in Theorem 3 also can be applied to Theorem 4 .

Remark 8. If $(X, \rho)$ is a metric space and for every $a \in X$ and every $\epsilon>0$ there exist $N \in \mathbb{N}$ and $\delta>0$ such that for every $n \geq N$ and every $x \in X$ fulfilling $\rho(x, a)<\delta$ we have $\left|\phi_{n}(x)-\phi_{n}(a)\right|<\epsilon$, then the function $\Phi$ is continuous. Indeed, for $a \in X$ and $\epsilon>0$ we get

$$
-\epsilon<\phi_{n+N}(x)-\phi_{n+N}(a)<\epsilon,
$$

for every $n \in \mathbb{N}$ and $x \in X$ satisfying $\rho(x, a)<\delta$. Hence,

$$
-\epsilon<\Phi(x)-\Phi(a)<\epsilon,
$$

for $x \in X$ fulfilling $\rho(x, a)<\delta$.

Remark 9. If functions $\phi_{n}$ satisfy the Lipschitz condition with a constant $\alpha$, then the fact that LIM is a continuous functional with a norm equal to 1 implies that the function $\Phi$ satisfies the Lipschitz condition with a constant $\alpha$.

We end the paper with the subsequent very simple observation showing one more application of the Banach limit.

Theorem 5. Let $0 \notin g(X), G_{n}$ be given by $(29), \phi: X \rightarrow \mathbb{R}$ and

$$
\varepsilon_{n}(x):=\frac{\phi\left(f^{n}(x)\right)}{G_{n}(x)}-\phi(x)-\sum_{i=0}^{n-1} \frac{h\left(f^{i}(x)\right)}{G_{i+1}(x)}, \quad x \in X, n \in \mathbb{N} .
$$

Assume that

$$
\iota(x):=\liminf _{n \rightarrow \infty} \varepsilon_{n}(x)>-\infty, \quad \sigma(x):=\limsup _{n \rightarrow \infty} \varepsilon_{n}(x)<\infty, \quad x \in X .
$$

Then there is a solution $\Phi: X \rightarrow \mathbb{R}$ of Eq. (3) such that

$$
\iota(x) \leq \Phi(x)-\phi(x) \leq \sigma(x), \quad x \in X .
$$

Proof. Write $\gamma_{k}(x):=\sup _{n \in \mathbb{N}} \varepsilon_{n+k}(x)$ and $\kappa_{k}(x):=\inf _{n \in \mathbb{N}} \varepsilon_{n+k}(x)$ for $x \in X$ and $k \in \mathbb{N}_{0}$. By the assumption

$$
\kappa_{k}(x) \leq \frac{\phi\left(f^{n+k}(x)\right)}{G_{n+k}(x)}-\sum_{i=0}^{n+k-1} \frac{h\left(f^{i}(x)\right)}{G_{i+1}(x)}-\phi(x) \leq \gamma_{k}(x), \quad x \in X, n \in \mathbb{N}, k \in \mathbb{N}_{0},
$$

which means that the sequence

$$
\phi_{n+k}(x):=\frac{\phi\left(f^{n+k}(x)\right)}{G_{n+k}(x)}-\sum_{i=0}^{n+k-1} \frac{h\left(f^{i}(x)\right)}{G_{i+1}(x)}, \quad n \in \mathbb{N},
$$


is bounded for every $x \in X$ and $k \in \mathbb{N}_{0}$. Define $\Phi: X \rightarrow \mathbb{R}$ by

$$
\Phi(x)=\operatorname{LIM}\left(\left(\phi_{n}(x)\right)_{n \in \mathbb{N}}\right), \quad x \in X .
$$

Since $\operatorname{LIM}\left(\left(\phi_{n}(x)\right)_{n \in \mathbb{N}}\right)=\operatorname{LIM}\left(\left(\phi_{n+k}(x)\right)_{n \in \mathbb{N}}\right)$ for all $x \in X$ and $k \in \mathbb{N}$ [see (1)], we have

$$
\kappa_{k}(x)+\phi(x) \leq \Phi(x) \leq \phi(x)+\gamma_{k}(x), \quad x \in X, k \in \mathbb{N}_{0},
$$

and consequently

$$
\iota(x)=\sup _{k \in \mathbb{N}_{0}} \kappa_{k}(x) \leq \Phi(x)-\phi(x) \leq \inf _{k \in \mathbb{N}_{0}} \gamma_{k}(x)=\sigma(x), \quad x \in X .
$$

In the same way as at the end of the proof of Theorem 4 we show that $\Phi$ is a solution to Eq. (3). This completes the proof.

Open Access. This article is licensed under a Creative Commons Attribution 4.0 International License, which permits use, sharing, adaptation, distribution and reproduction in any medium or format, as long as you give appropriate credit to the original author(s) and the source, provide a link to the Creative Commons licence, and indicate if changes were made. The images or other third party material in this article are included in the article's Creative Commons licence, unless indicated otherwise in a credit line to the material. If material is not included in the article's Creative Commons licence and your intended use is not permitted by statutory regulation or exceeds the permitted use, you will need to obtain permission directly from the copyright holder. To view a copy of this licence, visit http://creativecommons.org/ licenses/by/4.0/.

\section{References}

[1] Agarwal, R.P., Xu, B., Zhang, W.: Stability of functional equations in single variable. J. Math. Anal. Appl. 288, 852-869 (2003)

[2] Badora, R.: Report of meeting. Ann. Math. Sil. 20, 88 (2006)

[3] Badora, R.: Report of Meeting. Aequ. Math. 79, 175 (2010)

[4] Baker, J.A.: The stability of certain functional equations. Proc. Am. Math. Soc. 112, 729-732 (1991)

[5] Brillouët-Belluot, N., Brzdęk, J., Ciepliñski, K.: On some recent developments in Ulam's type stability. Abstr. Appl. Anal. 2012, 41 (2012). (Art. ID 716936)

[6] Brydak, D.: On the stability of the functional equation $\varphi[f(x)]=g(x) \varphi(x)+$ $F(x)$. Proc. Am. Math. Soc. 26, 455-460 (1970)

[7] Brzdęk, J., Ciepliński, K., Leśniak, Z.: On Ulam's type stability of the linear equation and related issues. Discrete Dyn. Nat. Soc. 2014, 14 (2014). (Art. ID 536791)

[8] Brzdęk, J., Popa, D., Raşa, I., Xu, B.: Ulam Stability of Operators. Academic Press, Oxford (2018) 
[9] Brzdęk, J., Popa, D., Xu, B.: Remarks on stability and nonstability of the linear functional equation of the first order. Appl. Math. Comput. 238, 141-148 (2014)

[10] Forti, G.L.: Hyers-Ulam stability of functional equations in several variables. Aequ. Math. 50, 143-190 (1995)

[11] Hyers, D.H., Isac, G., Rassias, ThM: Stability of Functional Equations in Several Variables. Birkhäuser, Basel (1998)

[12] Jung, S.-M.: Hyers-Ulam-Rassias stability of functional equations in nonlinear analysis. Springer Optimization and Its Applications, vol. 48. Springer, New York (2011)

[13] Kim, G.H.: On the stability of generalized gamma functional equation. Int. J. Math. Math. Sci. 23, 513-520 (2000)

[14] Kuczma, M.: Functional Equations in a Single Variable. PWN-Polish Scientific Publishers, Warszawa (1968)

[15] Kuczma, M., Choczewski, B., Ger, R.: Iterative Functional Equations. Encyclopedia of Mathematics and its Applications, vol. 32. Cambridge University Press, Cambridge (1990)

[16] Sofi, M.A.: Banach limits: some new thoughts and perspectives. J. Anal. (2019). https://doi.org/10.1007/s41478-019-00184-2

[17] Trif, T.: On the stability of a general gamma-type functional equation. Publ. Math. Debrecen 60, 47-61 (2002)

[18] Turdza, E.: On the stability of the functional equation $\varphi[f(x)]=g(x) \varphi(x)+$ $F(x)$. Proc. Am. Math. Soc. 30, 484-486 (1971)

[19] Ulam, S.M.: A collection of mathematical problems. In: Interscience Tract 8 (= Problems in Modern Mathematics, Science Edition), Interscience, New York (1960) (1964)

Roman Badora

Institute of Mathematics

University of Silesia

Bankowa 14

40-007 Katowice

Poland

e-mail: roman.badora@us.edu.pl

Janusz Brzdęk

Faculty of Applied Mathematics

AGH University of Science and Technology

Mickiewicza 30

30-059 Kraków

Poland

e-mail: brzdek@agh.edu.pl 
Received: August 31, 2020.

Accepted: February 7, 2021.

Publisher's Note Springer Nature remains neutral with regard to jurisdictional claims in published maps and institutional affiliations. 